\title{
Aprender a definir en Matemáticas: estudio desde una perspectiva sociocultural
}

\section{Learning to define in Mathematics: a study from a sociocultural perspective}

\author{
José María Gavilán Izquierdo, Gloria Sánchez-Matamoros, Isabel Escudero \\ Departamento de Didáctica de las Matemáticas \\ Universidad de Sevilla \\ gavilan@us.es, gsanchezmatamoros@us.es, escudero@us.es
}

RESUMEN - Los procesos de construcción de conocimiento matemático, como definir, son clave en la actividad de "hacer matemáticas». La caracterización de su aprendizaje es un tema relevante en el campo de investigación en educación matemática. En este trabajo, adoptando una perspectiva sociocultural, a través de un análisis inductivo, hemos identificado diferentes situaciones de aprendizaje matemático. Dichas situaciones se caracterizan a través de las herramientas teóricas procedentes de la perspectiva sociocultural cuando grupos de estudiantes resuelven tareas en un contexto geométrico. Los resultados muestran que determinadas rutinas juegan un papel relevante en los procedimientos de cambio en el discurso matemático.

PALABRAS CLAVE: definir; perspectiva sociocultural; procedimientos de cambio discursivo; situaciones de aprendizaje.

ABSTRACT - The process of building mathematical knowledge, as defined, are key activity of «doing mathematics». The characterization of their learning is an important topic in the field of research in mathematics education. In this paper we adopt a sociocultural perspective, through an inductive analysis identified different situations of learning. Such situations are characterized by the theoretical tools from the sociocultural perspective when groups of students solve tasks in a geometric context. The results show that certain routines play an important role in the processes of change in mathematical discourse.

KEYWORDS: defining; sociocultural perspective; procedures for discursive change; situations of learning. 


\section{INTRODUCCIÓN}

La caracterización del aprendizaje matemático es un tema relevante en el campo de investigación en educación matemática. Recientemente se han incorporado perspectivas socioculturales para abordar dicha problemática (en el ámbito internacional, por ejemplo, Lave y Wenger, 1991; Lerman, 1996; Sfard, 2008; Cobb, Gresalfi y Liao, 2009; y en el ámbito español, por ejemplo, Llinares, 2000; Gavilán, García y Llinares, 2007; Planas y Civil, 2010). Las teorías socioculturales en la educación, que surgen con la influencia de la obra de Vygostki (1962), sostienen que el proceso de desarrollo cognitivo individual no es independiente o autónomo de los procesos socioculturales, ni de los procesos educacionales en particular. El papel de la interacción social con los otros tiene una gran importancia en el desarrollo del individuo. Además de las relaciones sociales, la mediación a través de los instrumentos (no solo físicos sino también psicológicos como lenguaje, escritura, libros, ordenadores, etc.) permite dicho desarrollo (Wenger, 1998).

En estas perspectivas hay una concepción del conocimiento matemático como construcción social y cultural (Lerman, 2000, 2006) y muchos trabajos centran la atención en el análisis de los procesos por los cuales se produce dicha construcción social. En esta construcción social tiene un papel relevante el discurso, que tiene que ver con compartir significados y modos de interpretar cómo actuar en ciertos contextos. Para Sfard (2006) las matemáticas son un tipo especial de discurso y aprender matemáticas significa cambiar el discurso matemático.

La presencia de investigaciones que adoptan perspectivas socioculturales es cada vez más relevante en nuestro país. El trabajo de Cobo y Fortuny (2000) utiliza elementos del análisis del discurso para investigar sobre las interacciones de los estudiantes cuando resuelven problemas de matemáticas. Por su parte, Planas y Civil (2010) abordan el estudio de los procesos discursivos en clase de matemáticas y cómo afectan al aprendizaje. En esta línea, Chico y Planas (2011) señalan la importancia de la interacción entre los estudiantes para que se produzca aprendizaje matemático. En este trabajo, el discurso se considera como elemento relevante para la manifestación del aprendizaje producido mediante las interacciones, con el objetivo de «conseguir relacionar avances en el aprendizaje matemático con interacciones entre estudiantes caracterizadas con indicadores [...] (refutación, validación, cuestionamiento y paráfrasis)» (pp. 320 y 323). Más recientemente, Morera, Planas y Fortuny (2013) y Planas y Chico (2013), para investigar el aprendizaje que se produce en las discusiones en gran grupo, cuando resuelven tareas en clase de matemáticas en distintos contextos, introducen herramientas analíticas para identificar y caracterizar las oportunidades de aprendizaje matemático que se producen.

Por otro lado, Llinares (2000) plantea el análisis de la práctica del profesor utilizando la noción de instrumento de la práctica, su uso y propósito del uso de este, en el análisis del discurso. En Gavilán et al. (2007), se hace uso de dichas nociones teóricas en el análisis de prácticas del profesor en el contexto de enseñanza de la derivada.

Por su parte, Sánchez y García $(2011,2013)$ han tratado de investigar la existencia de relaciones entre normas sociomatemáticas y normas matemáticas en diferentes niveles académicos. En su estudio han podido identificar diferentes conflictos comognitivos (Sfard, 2008) que surgen de la confrontación entre normas sociomatemáticas y normas matemáticas ligadas a la definición.

Distintas investigaciones, en el ámbito internacional, se apoyan en la perspectiva comognitiva (commognitive en el original) de Sfard (2007). En el estudio de Bowers y Stephens (2011) se aborda la problemática de la incorporación de la tecnología en cursos de formación de profesores de matemáticas, donde un papel relevante lo juega el uso de las herramientas apropiadas (tanto conceptuales -símbolos- como tecnológicas) como parte del proceso comognitivo de aprendizaje. Güçler (2013) indaga sobre la coherencia entre los discursos de los estudiantes y el profesor cuando abordan el concepto de límites de funciones a nivel universitario. En sus resultados detecta algunas dificultades de comunicación entre estudiantes y profesor con relación a las diferentes narrativas aceptadas. 
Por otro lado, considerando los procesos de construcción del conocimiento matemático, desde un enfoque sociocultural, Rasmussen, Zandieh, King y Teppo (2005) y Zandieh y Rasmussen (2010) elaboran un marco integrado, en el sentido de caracterizar de forma conjunta las distintas actividades matemáticas avanzadas propias del pensamiento matemático, tales como simbolizar, algoritmizar y definir. Para estos investigadores, el aprendizaje de las matemáticas significa participar en diferentes tipos de prácticas matemáticas. Estos autores estructuran el rol de definir en el progreso del estudiante desde razonamientos informales a maneras más formales de razonamiento.

Asumimos que los procesos de construcción de conocimiento matemático, tales como definir, probar y modelar, son elementos clave en el aprendizaje del conocimiento matemático, con una configuración compleja, multidimensional y universal (Sánchez, García, Escudero, Gavilán y SánchezMatamoros, 2008).

Por otro lado, algunas investigaciones (García, Sánchez, Escudero y Llinares, 2003; García, Sánchez y Escudero, 2007), desde perspectivas socioculturales del aprendizaje, proponen un marco teórico y metodológico para la identificación y caracterización de diferentes niveles de aprendizaje en grupos de estudiantes. En su investigación, estos autores conciben el aprendizaje a partir del uso de herramientas conceptuales, entendidas como aquellas ideas teóricas generadas desde las investigaciones sobre educación matemática. En trabajos anteriores (Sánchez-Matamoros, Escudero, Gavilán, 2011; Gavilán, Sánchez-Matamoros y Escudero, 2012; Escudero, Gavilán y Sánchez-Matamoros, en prensa), hemos caracterizado cambios en el discurso matemático de grupos de estudiantes, como indicador del aprendizaje matemático desde una perspectiva sociocultural (Sfard, 2007 y 2008). En este trabajo, tratamos de avanzar en la caracterización de este aprendizaje matemático mediante la identificación de diferentes situaciones de aprendizaje matemático, determinadas por las características de los cambios que se producen en el discurso en grupos de estudiantes.

En lo que sigue, en primer lugar presentamos el marco teórico en el que enmarcamos este trabajo, que guía el diseño metodológico utilizado. Mostraremos algunos ejemplos del funcionamiento del esquema metodológico para identificar y caracterizar las diferentes situaciones de aprendizaje matemático. Por último, reflexionaremos sobre los resultados obtenidos y el potencial que puede tener para futuras investigaciones.

\section{MARCO TEÓRICO}

En la elaboración del marco teórico hemos tenido en cuenta: el aprendizaje desde una perspectiva sociocultural y el proceso de definir, que pasamos a describir a continuación.

\section{El aprendizaje matemático desde una perspectiva sociocultural}

Nuestra investigación está basada en la aproximación sociocultural de Sfard (2006, 2007 y 2008) en la que se considera que el aprendizaje viene dado por los cambios en el discurso, distinguiendo dos tipos de discurso, el coloquial y el especializado (por ejemplo, el discurso matemático). Para caracterizar el discurso matemático y su cambio, Sfard (2007) propone el uso de cuatro características discursivas: palabras matemáticas, mediadores visuales, narrativas asumidas y rutinas, que describimos a continuación:

Palabras matemáticas (mathematical words): aquellas utilizadas en el discurso matemático, incluyendo términos no propiamente matemáticos con significado matemático y utilidad matemática. 
Mediadores visuales (visual mediators): entendidos como los medios con los que los participantes del discurso identifican los objetos de los que están hablando y coordinan su comunicación. En los discursos matemáticos, entre los mediadores visuales se incluyen los símbolos, gráficos, etc. Narrativas asumidas (endorsed narratives): entendidas como cualquier texto hablado o escrito que da una descripción de los objetos, de relaciones entre objetos o de actividades con los objetos y que está sujeto a la aceptación o rechazo del colectivo. Se utiliza el término narrativas asumidaslaceptadas para referirse a las que se etiquetan como verdaderas por una comunidad dada en un momento determinado.

Rutinas (routines): son patrones repetitivos bien definidos en las acciones de los interlocutores, característicos de un discurso dado. Las regularidades específicamente matemáticas pueden ser identificadas si uno ve el uso de las palabras matemáticas y los mediadores matemáticos o sigue el proceso de crear y fundamentar narrativas asumidas o rechazar otras asumidas previamente. Las rutinas pueden ser de naturaleza algorítmica.

\section{La definición matemática y el proceso de definir}

El papel importante que tiene la definición matemática y el proceso de definir en el conocimiento de las matemáticas ha sido destacado por distintos investigadores en las últimas décadas (Vinner y Hershkowitz, 1980; Tall y Vinner, 1981; Hershkowitz 1990; Mariotti y Fischbein, 1997; MamonaDowns y Downs, 2002). Más recientemente, Zazkis y Leikin (2008) consideran que las definiciones de conceptos matemáticos, las estructuras subyacentes de las definiciones y el proceso de definir deben ser componentes fundamentales del conocimiento de la materia de los profesores de matemáticas. Además, como señalan Marioti y Fischbein (1997), aprender a definir es un problema básico en la educación matemática. Ahora bien, no se trata de un proceso falto de dificultad para los estudiantes, como señalan Rasmusen et al. (2005): "crear y usar definiciones matemáticas versus "definiciones de cada día”, es una actividad esencial y a menudo difícil para los estudiantes, incluso para aquellos que están en cursos superiores» (p. 65).

Según Mariotti y Fischbein (1997: 220) definir un concepto es «dar un listado de propiedades que lo caracterizan». En nuestro trabajo, con relación a definir/definición, consideramos definir como el proceso para llegar a establecer una definición, teniendo en cuenta que esta última prescribe el significado de una palabra o frase de forma específica en términos de una lista de características que tienen que ser todas verdaderas (Sánchez et al., 2008).

En resumen, en este trabajo abordaremos el proceso de definir en grupos de estudiantes cuando resuelven una tarea en un contexto geométrico, haciendo uso de las herramientas teóricas (palabras matemáticas, mediadores visuales, narrativas asumidas y rutinas) propuestas por Sfard (2007 y 2008). El objetivo de este trabajo es caracterizar el aprendizaje matemático mediante la identificación de diferentes situaciones de aprendizaje matemático, determinado por las características de los cambios que se producen en el discurso en grupos de estudiantes.

\section{METODOLOGÍA}

\section{Contexto y participantes}

La investigación se ha desarrollado en un contexto de formación inicial de profesores de Educación Primaria de una universidad española y en un instituto de educación secundaria en el nivel de bachillerato.

Los participantes en el estudio fueron estudiantes de enseñanza no obligatoria, 38 estudiantes para profesor de Educación Primaria (edades entre 18 y 21 años) y 13 estudiantes de segundo curso de ba- 
chillerato (edades entre 16 y 18 años). Estos estudiantes participaron en el estudio de forma voluntaria y se organizaron en grupos de tres o cuatro según su criterio (los grupos los hemos denominado como G1... G15; los grupos G13, G14 y G15 corresponden a estudiantes de bachillerato). Así, vamos a considerar como sujetos de análisis grupos de estudiantes resolviendo tareas.

\section{Fuentes de datos}

Los datos de nuestro estudio consistían en las transcripciones de las grabaciones en audio de los diálogos verbales de grupos de estudiantes, mantenidos durante la sesión de trabajo al resolver una tarea, y en los documentos escritos que elaboraron los estudiantes para responder a las cuestiones propuestas en la tarea.

\section{La tarea}

Planteamos una tarea que aborda la definición matemática en un contexto geométrico, en particular la clasificación de paralelogramos, con el objetivo de que fuera un contenido con el que los estudiantes estuvieran familiarizados y no un elemento distractor del objetivo de la investigación.

La tarea se planteó por escrito a cada grupo de estudiantes, indicándoles que debían verbalizar sus ideas cuando fueran respondiendo a las diferentes preguntas planteadas. En ella (figura 1) se presentaban las figuras de tres cuadriláteros (cuadrado, rectángulo y rombo) y nueve preguntas secuenciadas de manera que en primer lugar se centraban en las figuras individualmente y después en las relaciones entre figuras. Con ello los grupos de estudiantes irían identificando aquellos elementos de las figuras (lados, ángulos interiores y exteriores entre lados, diagonales y ángulos entre diagonales...), características o propiedades de estas, que llevaran a definir los conceptos de cuadrado, rombo y rectángulo. Algunos ejemplos de estas preguntas fueron los siguientes: «¿Qué propiedades o características observas en cada una de estas figuras?»; “¿Hay características o propiedades comunes a las tres?»; «¿Puedes definir cada una de las figuras?», $\mathrm{y}$ « Puedes dar otra definición de cada una de las figuras?». La tarea se realizó en una sesión de dos horas de duración.

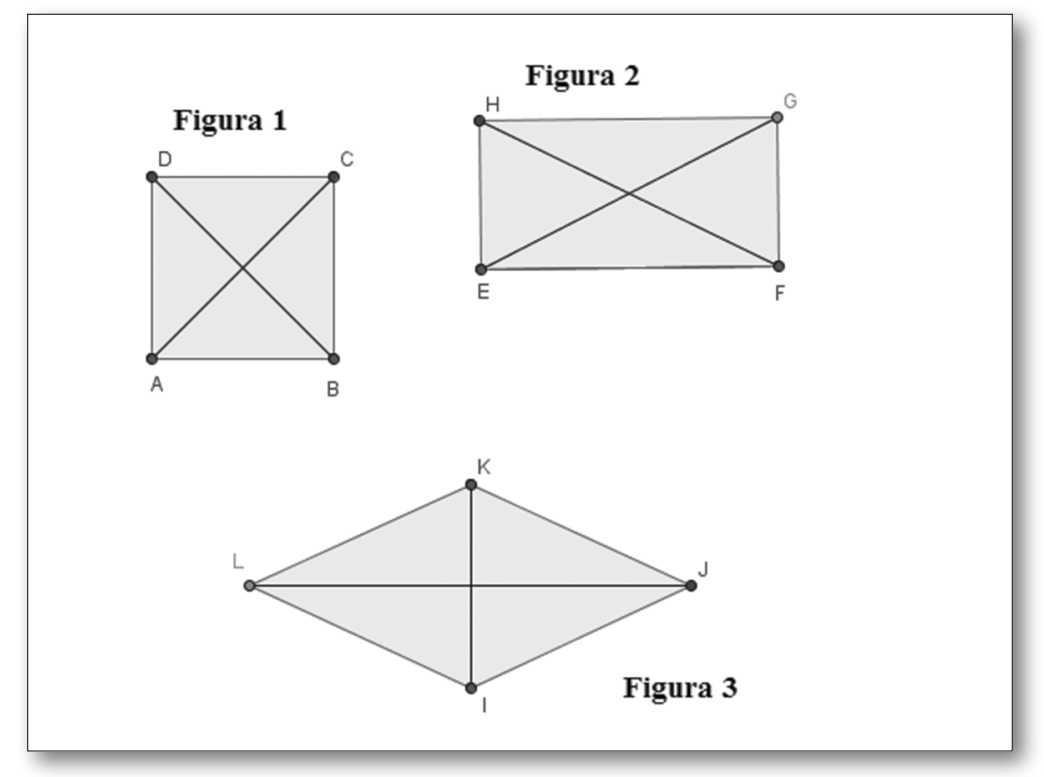

Fig. 1. Figuras de cuadriláteros presentadas en la tarea. 


\section{Análisis}

El procedimiento de análisis seguido ha sido de tipo inductivo, a partir de las características de los procedimientos de cambio identificados en el discurso matemático, a través de las herramientas teóricas (palabras matemáticas, mediadores visuales, narrativas asumidas y rutinas) y de relaciones entre dichas herramientas. Dichos procedimientos de cambios (unidad de análisis) (Gavilán et al., 2012; Escudero et al., en prensa) se caracterizaron mediante la identificación de los siguientes elementos:

- Narrativas asumidas y secuencias entre estas.

- Rutinas identificadas y papel que desempeñan en la secuencia de narrativas.

- Cambios producidos en el uso de palabras matemáticas y mediadores visuales.

Las respuestas de los distintos grupos de estudiantes fueron categorizadas en función de si en el discurso matemático de cada grupo de estudiantes se habían identificado (o no) cambios. En el caso de que se identificara algún cambio, observábamos el tipo de cambio que se había producido según los elementos mencionados anteriormente.

Los cambios en el discurso de los grupos de estudiantes podían producirse en una o varias etapas. Consideramos que se produce un cambio en el discurso de una etapa cuando se produce un cambio de una narrativa asumida a otra, independientemente de que haya, o no, rechazo de la primera narrativa. Los cambios en el discurso en varias etapas son aquellos en los que se producen al menos dos cambios, pasando en cada uno de ellos de una narrativa asumida a otra distinta, independientemente de que haya, o no, rechazo de la narrativa anterior (Escudero et al., en prensa).

A partir de los elementos de análisis considerados encontramos cuatro categorías de grupos de estudiantes que comparten rasgos comunes:

- Grupos de estudiantes en los que no se producen cambios en el discurso matemático.

- Grupos de estudiantes en los que se produce un cambio en el discurso matemático en una etapa, caracterizado por el paso de una narrativa asumida a otra narrativa asumida distinta, a través del uso de determinadas rutinas y del cambio en el significado de las palabras matemáticas y/o en los mediadores visuales que intervienen en el discurso. La aceptación de la nueva narrativa conlleva el rechazo de la primera.

- Grupos de estudiantes en los que se producen cambios en el discurso en varias etapas, caracterizadas por el paso, en cada una de ellas, de una narrativa asumida a otra narrativa asumida distinta, a través del uso de determinadas rutinas y del cambio en el significado de las palabras matemáticas y/o en los mediadores visuales que intervienen en el discurso. La aceptación de cada nueva narrativa conlleva el rechazo de la anterior.

- Grupos de estudiantes en los que se producen cambios en el discurso en varias etapas, donde los cambios de narrativas no implican, necesariamente, el rechazo de otras. Se caracteriza porque los grupos disponen de un repertorio de varias narrativas asumidas con las que afrontar situaciones problemáticas.

\section{RESULTADOS}

Las categorías anteriormente descritas nos permitieron identificar diferentes situaciones de aprendizaje en los procedimientos de cambios en el discurso matemático. A continuación, mostramos las características de cada una de estas situaciones identificadas, a través de un ejemplo de cada situación. 


\section{Situación de invariancia en el discurso matemático en definir}

Esta situación se caracteriza porque no hay variación en el discurso matemático, ya que no se producen cambios en las herramientas discursivas, solo se asume una narrativa, se identifican una o varias rutinas y se detectan dificultades en dotar de significado algunas palabras matemáticas. Solo se utilizan como mediador visual las figuras proporcionadas en la tarea.

Un ejemplo de esta situación lo encontramos en el grupo 2 (G2). Este grupo, cuando trata de definir, hace uso de las características matemáticas de los objetos, a través de un listado de estas (protocolo 2.1). Esto constituye una evidencia empírica de que el grupo asume la narrativa «definir es dar una lista de características de los objetos que se pretenden definir» (narrativa Listado de características).

Protocolo 2.1:

457 A4: pregunta seis, definir cada una de las figuras .......

460 A2: no, es que, un polígono regular, un polígono regular de cuatro lados

461 A4: figura uno, esperaros un momento, por favor

462 A2: el uno es polígono regular

463 A3: de cuatro lados

$464 \mathrm{~A} 3$ : es un polígono regular cerrado

465 A2: de cuatro lados

466 A4: iguales

.......

472 A2: polígono cerrado de cuatro lados, diagonales perpendiculares y diferentes

$473 \mathrm{~A} 3$ : cuatro lados iguales y de $90^{\circ}$, son ángulos de $90^{\circ}$

Asumir esta narrativa conlleva, en este grupo, hacer uso de dos rutinas. En la primera, los estudiantes identifican propiedades o características de cada una de las figuras implicadas, fijando la atención directamente en los elementos geométricos básicos de dichas figuras (que han sido identificados y enunciados previamente, ya que se demandaba al inicio de la tarea), como puede observarse en los protocolos 2.2 y 2.3, en los que el grupo de estudiantes trata de dar significado a lo que se consideran características o propiedades de una figura. En particular, en el protocolo 2.3 se da significado a característica de una figura en contraposición a nombrarla.

Protocolo 2.2:

98 A1: es un cuadrado

$99 \mathrm{~A} 1$ : respecto a los lados la característica es que es un polígono cerrado de cuatro lados

100 A2: iguales, por tanto se llama cuadrado

Protocolo 2.3:

134 A3: una característica no es lo que es, es un cuadrado

135 A1: una característica del

136 A2: la característica del ángulo es que mide $90^{\circ}$

La estructura de esta rutina (rutina Identificar caracteristicas) es la siguiente: miran la figura, identifican elementos relevantes de la figura, enuncian propiedades de los elementos relevantes de la figura.

En la segunda rutina, identifican características comunes y no comunes a varias figuras. Una evidencia empírica la encontramos en el protocolo 2.4, en el que se observa cómo los estudiantes centran su atención en extraer las características comunes de las figuras (esto se hace especialmente evidente en las líneas 375 y 376, donde destacan la propiedad de que el cuadrado y el rombo tienen sus lados iguales). La estructura de esta rutina (rutina Identificar caracteristicas comunes) es: miran una figura, 
identifican una propiedad o característica y comprueban si otra figura tiene esa característica. En ambas rutinas el grupo utiliza como mediador visual las figuras del enunciado de la tarea, sin incorporar otros mediadores visuales.

Además, en dicho protocolo se pone de manifiesto que los estudiantes de este grupo (G2) tienen dificultad en dotar de significado la idea de "característica de una figura», ya que, por un lado, hablan de características de lados y, por otro, de que la fórmula del perímetro también es una característica. Esto evidencia que este grupo tiene dificultades en el uso de determinadas palabras matemáticas (característica de la figura) necesarias para definir. Este hecho puede ser una causa que impida que se produzcan cambios de narrativas asumidas.

Protocolo 2.4:

351 varios: estamos con las figuras uno y tres

352 el perímetro

353 A2: el perímetro se calcula de la misma forma

.......

371 A2: bueno, tiene los ángulos centrales, forman $90^{\circ}$, pero no sé, claro, que son

372 perpendiculares

373 A3: y que son rectángulos, bueno, lo ponemos a tu manera, los ángulos son centrales

374 A2: yo estoy poniendo perpendicularmente entre paréntesis, los centrales

375 A3: ya está, ¿̇no? Ah, bueno, y que ellos, los lados, tienen la misma medida

376 A2: en el cuadrado miden todos 3 y en el rombo miden todos 6

377 A3: todos los lados miden iguales

En resumen, en el discurso de este grupo se asume que definir es dar un listado de características. En relación con las palabras matemáticas utilizadas en el discurso del grupo, podemos decir que no se produce ni ampliación de vocabulario ni cambio en el significado de las palabras matemáticas, mostrando dificultades en dar significado a algunas de ellas. Respecto a los mediadores visuales, en el discurso del grupo no se pone de manifiesto la necesidad de utilizar otros mediadores visuales distintos de los proporcionados en la tarea. El hecho de que no se produzca ningún cambio de narrativa, ni de otros tipos de herramientas discursivas, nos ha llevado a considerar que lo que caracteriza a este grupo es que no se producen variaciones en el discurso matemático (invariancia en el discurso).

\section{Situación de un cambio en el discurso matemático en definir con abandono de narrativas}

Esta situación se caracteriza por un cambio en el discurso matemático de una narrativa asumida a otra narrativa asumida distinta, a través del uso de determinadas rutinas fundamentalmente, aunque también conlleva cambios en el significado de las palabras matemáticas y/o en los mediadores visuales que intervienen en el discurso. La aceptación de la nueva narrativa conlleva el rechazo de la primera.

Un ejemplo de esta situación lo encontramos en el grupo 9 (G9). Este grupo de estudiantes comienza considerando que es suficiente con poner un nombre para definir un concepto (protocolo 9.1, líneas 526-529), lo que es una evidencia empírica de que el grupo asume la narrativa «definir es etiquetar, poner un nombre a una clase, más o menos amplia, de objetos» (narrativa Etiquetar).

Protocolo 9.1

525 A1: Venga, definid cada una de las figuras

526 A3: La figura uno es un cuadrado

527 A1: Es un cuadrado...

528 A2: Cuadrado, rectángulo y rombo

529 A1: Bueno, pero habrá que definir algo más, ¿̇no? 
530 A2: No, ¿̨no?

531 A3: Vamos a ver, Elena, es un cuadrado, ¿tú que le vas a decir a

532 un nińo chico?

A lo largo de la resolución de la tarea se pone de manifiesto un cambio en las narrativas asumidas por los estudiantes del grupo. El grupo asume que para definir es necesario dar un listado de características de las figuras, narrativa Listado de características (protocolo 9.2).

Protocolo 9.2:

559 A3: Ponlo, es un cuadrado que tiene cuatro lados iguales que...

560 A2: Claro

561 A1: Es una figura compuesta por cuatro lados iguales, paralelos

562 dos a dos, ¿¿no?

Este cambio entre las narrativas asumidas se produce mediante cambios en el significado de algunas palabras matemáticas, en los mediadores visuales y por el uso de la rutina Identificar características (rutina que se observa cuando están dando las características de las distintas figuras, por ejemplo para el cuadrado "cuatro vértices, cuatro lados iguales, cuatro ángulos rectos...»). El carácter de rutina de esta manera de proceder es debido a que la hemos identificado en el discurso del grupo en varios momentos, como puede observarse en el protocolo 9.3.

Protocolo 9.3

62 A1: a ver, primera figura

63 A3: por allí están escribiendo desde luego

$64 \mathrm{A1}$ : cuatro vértices, cuatro lados

65 A3: dos diagonales

66 A2: cuatro lados iguales

67 A3: ah, eso sí. Cuatro vértices equidistantes entre sí

68 A2: y cuatro ángulos rectos. Y vértices no hay, ¿̇no? Ah, sí, sí

.......

173 A2: bueno, esto es un rectángulo

174 A1: cuatro vértices

175 A3: cuatro lados iguales dos a dos

176 A2: cuatro ángulos rectos

177 A3: dos diagonales

El cambio, aunque venga propiciado principalmente por el uso de la rutina anterior, también conlleva cambios en el significado de algunas palabras matemáticas y en los mediadores visuales que intervienen en el discurso. Así, respecto a las palabras matemáticas relacionadas con definir pasan de ser etiquetas (líneas 526-528 del protocolo 9.1) a significar un conjunto de características (líneas 561-562 del protocolo 9.2). Con relación al uso de mediadores visuales, los estudiantes de este grupo, en su afán por dar definiciones a través de las características de las figuras que se están definiendo, intentan buscar ejemplos (mediante dibujos de figuras geométricas) en los que se destaquen características no comunes (aunque sí lo sean), como es el caso en el que se discute que dos figuras (cuadrado y rectángulo) puedan tener una característica común (ángulos entre lados de 90) (protocolo 9.4).

Protocolo 9.4

198 A1: bueno, venga, pongo cuatro ángulos rectos. Pero es que es

199 imposible que esto tenga cuatro ángulos rectos y este cuatro

200 ángulos rectos, porque es diferente

201 A2: sí, sí 
202 A3: que si no, de qué, Elena. Esto es recto, esto es recto, esto es

203 recto y esto es recto

204 A2: lo único que diferencia es que están más alargados

205 A3: si no fuera recto sería que estuvieran así

206 A2: son cuadrados y son rectángulos

En resumen, en el discurso de este grupo se produce un cambio de una narrativa asumida (narrativa Etiquetar) a una nueva narrativa (narrativa Listado de caracteristicas), con abandono de la primera narrativa a través del uso de la rutina Identificar características y la modificación de los significados de palabras matemáticas involucradas en el discurso, junto con el uso de nuevos mediadores visuales.

\section{Situación de varios cambios secuenciados en el discurso matemático en definir, con aban- dono de narrativas}

Esta situación se caracteriza por cambios en el discurso que se producen en varias etapas, del mismo tipo que las identificadas en la situación anterior, de tal forma que la aceptación de una nueva narrativa conlleva el rechazo de la anterior. Estos cambios, aunque caracterizados fundamentalmente por el uso de determinadas rutinas, también conllevan cambios en el significado de las palabras matemáticas y en los mediadores visuales que intervienen en el discurso.

Un ejemplo de esta situación lo encontramos en el grupo 4 (G4), en el que el cambio en el discurso se produce en dos etapas. En la primera etapa, el grupo comienza asumiendo la narrativa Etiquetar (protocolo 4.1), posteriormente a lo largo de la resolución de la tarea se va a producir un cambio de discurso para asumir una nueva narrativa, narrativa Listado de características (protocolo 4.2), rechazando la primera.

Protocolo 4.1:

530 A1: es decir, un cuadrado

531 A2: pero exactamente

532 A4: cuadrado, rectángulo

533 [Risas]

534 A2: es que yo creo que aquí lo que pide es el nombre y ya está

Protocolo 4.2:

$580 \mathrm{~A} 1:$ es que si son cuatro lados iguales ya...

581 A2: es que ya estamos hablando de características

582 A3: polígono regular de cuatro lados iguales

Este cambio entre narrativas asumidas se produce a través del uso de la rutina Identificar caracteristicas. Esta rutina, como conjunto de acciones, se aprecia en el discurso del grupo en distintos momentos (protocolos 4.3 y 4.4), vinculados a los distintos momentos en los que el grupo de estudiantes está identificando los elementos básicos de las figuras geométricas (número de lados, número de diagonales, ángulos que se forman) y las relaciones entre dichos elementos (igualdad de lados, igualdad de ángulos, paralelismo de lados, medida de ángulos).

Protocolo 4.3:

8 A1: ¿el número de lados?

9 A3: el número de diagonales, ¿̨no?

10 A2: ¿cuáles son los elementos básicos de cada una de estas figuras?

$11 \mathrm{~A} 3$ : ¿el ángulo? 
12 A2: el ángulo que forman

13 A1: voy a apuntarlo, ¿vale?

14 A2: el número de lados, el ángulo que forman

Protocolo 4.4:

90 A2: ahora es cuando hay que decir que en la $a$

$91 \mathrm{~A} 1$ : los lados iguales y por lo tanto

92 A2: lados iguales y ángulos iguales. Lados iguales y paralelos dos a dos

93 A1: sí

94 A2: y ángulos rectos también. Aquí, lados iguales y paralelos [...]

De forma análoga a lo indicado en la situación anterior, en este grupo el cambio en el discurso producido al pasar de una narrativa asumida a otra, aunque caracterizado principalmente por la rutina anterior, también conlleva cambios en el significado de algunas palabras matemáticas. Por ejemplo, en un primer momento, la palabra cuadrado se utiliza para etiquetar una determinada forma geométrica en el sentido de «la figura es un cuadrado» (línea 530 del protocolo 4.1); sin embargo, cuando asumen la narrativa Listado de características, el mismo término es una palabra que se utiliza para designar una figura geométrica que cumple unas determinadas características (línea 582 del protocolo 4.2).

En la segunda etapa, el cambio en el discurso se produce cuando el grupo abandona la narrativa Listado de características y asume que "definir es dar una lista mínima de características de los objetos que se están definiendo" (narrativa Listado mínimo de características) (línea 628, protocolo 4.5). Asumir esta narrativa supone que en la definición no tiene por qué aparecer una lista exhaustiva de propiedades, es decir, lo que se observa es una tendencia a dar una definición con el atributo de la minimalidad.

Protocolo 4.5:

627 A4: a ver cuatro lados iguales con ángulos rectos

628 A1: [escribiendo] 4 lados iguales que forman ángulos rectos. ¿¿Algo más?

629 A4: yo creo que no

630 A1: ¿ponemos lo de las diagonales?

631 A2: es que estamos hablando de la definición, una definición de las figuras, no de las

632 características

Este cambio entre narrativas asumidas se produce cuando los estudiantes hacen uso de dos rutinas. Estas rutinas son: «Identificar relaciones (implicativas) entre características de los objetos» (rutina Identificar relaciones implicativas) y «Poner ejemplos y contraejemplos» (en adelante, rutina Poner ejemplos), que se observan cuando están estudiando que determinadas características implican otras. Aunque los estudiantes son conscientes de las relaciones entre propiedades (rutina Identificar relaciones implicativas) (líneas 40 y 46, protocolo 4.6) antes de empezar a dar definiciones, solo usan estas relaciones cuando empiezan a asumir la nueva narrativa Listado mínimo de características (línea 631, protocolo 4.5)

Protocolo 4.6:

$38 \mathrm{~A} 1$ : el ángulo que forman los lados y el ángulo que forman las diagonales, ¿̇dices tú?

39 A3: yo decía los de las diagonales

40 A2: sabiendo el ángulo que forman los lados sabes los ángulos que forman las diagonales, 41 que es la bisectriz

.......

46 A2: es que las diagonales y el ángulo de las diagonales va en función de los ángulos, del 47 ángulo que forman los lados y de la longitud de los lados 
Respecto a la rutina Poner ejemplos, podemos señalar que los estudiantes hacen uso de esta rutina cuando están indagando qué ocurre cuando se están eliminando características de la definición (que se deducen de las características o propiedades que se dejan en la definición) y el alcance que tiene la nueva definición para incluir otra figura. Una evidencia empírica puede observarse en el protocolo 4.7, en el que los estudiantes están debatiendo sobre la característica del rectángulo de «tener los lados iguales dos a dos», que puede extenderse a los cuadrados. Por tanto, estos últimos son un caso particular de los rectángulos; de esta manera el grupo de estudiantes está cambiando los significados vinculados a las palabras matemáticas cuadrado y rectángulo.

Protocolo 4.7:

660 A3: iguales dos a dos, yo no te dibujaría un cuadrado

661 A2: tú no me lo dibujarías, pero ¿podrías dibujármelo?

662 A3: no. Yo no te lo dibujaría

663 A1: pero sí podría ser

664 A2: pero podría

665 A1: sí, está dentro de la definición

........

670 A2: por eso mismo no te doy toda la información para que tú puedas darme dos

671 opciones diferentes de respuesta.

Por tanto, en esta segunda etapa, el uso conjunto de estas dos rutinas, Identificar relaciones implicativas y Poner ejemplos, facilita el cambio en el discurso, asumiendo el grupo la nueva narrativa Listado minimo de caracteristicas, después de rechazar la narrativa Listado de características.

En relación con los cambios en el significado de las palabras matemáticas que se produce en el discurso, podemos señalar que en el grupo se aprecian diferencias entre poner un listado de características y definir (línea 631, protocolo 4.5), es decir, para el grupo definir una figura no es listar todas sus características. En esta segunda etapa del cambio producido en el discurso, los mediadores visuales (dibujos realizados por los grupos de estudiantes durante el proceso de resolución de la tarea) juegan un papel relevante, que se pone especialmente de manifiesto en la última rutina.

En resumen, en el discurso de este grupo hemos identificado cambios en el discurso en dos etapas. En la primera, se produce el cambio de la narrativa asumida Etiquetar a la narrativa Listado de características. En la segunda, se da un cambio de la narrativa Listado de características a la nueva narrativa Listado minimo de características. En ambas etapas, la aceptación de una narrativa conlleva el rechazo o abandono de la anterior. Estos cambios han supuesto cambios en los significados de las palabras matemáticas y en los mediadores visuales, y se han llevado a cabo mediante el uso de diversas rutinas (Identificar características, Identificar relaciones implicativas y Poner ejemplos).

\section{Situación de varios cambios coordinados en el discurso matemático en definir}

Esta situación se caracteriza por cambios en el discurso que se producen en varias etapas, del mismo tipo que las identificadas en la situación de un cambio en el discurso matemático con abandono de narrativas, aunque en esta situación la aceptación de una nueva narrativa no conlleva siempre el rechazo de anteriores narrativas, conviviendo en el discurso del grupo distintas narrativas. Estos cambios, aunque caracterizados por el uso de determinadas rutinas, también conllevan cambios en el significado de las palabras matemáticas y la introducción de nuevas palabras matemáticas y de nuevos mediadores visuales que intervienen en el discurso.

Un ejemplo de esta situación lo encontramos en el grupo 3 (G3). Esta situación de aprendizaje se pone de manifiesto a través de un cambio en el discurso que se produce en dos etapas. En una primera etapa se pasa de la narrativa asumida Listado de características a una nueva narrativa asumida, Listado mínimo de características. 
El grupo comienza considerando que para definir es necesario dar un listado de propiedades o características matemáticas de los objetos que se están definiendo, es decir, se asume la narrativa Listado de características, y además hacen uso de otras palabras matemáticas cuando se refieren al cuadrado como un cuadrilátero (protocolo 3.1).

Protocolo 3.1:

155 A1: el cuadrado es una figura geométrica

156 A2: cuadrilátero, ¿no?

157 A1: yo iba a poner formada por 4 lados iguales, ¿no?

158 A2: formada por 4 lados, pon entre paréntesis cuadrilátero

159 A1: 4 lados iguales y 4 ángulos

..../...

165 A2: ... ahora, en el rectángulo pon que tiene lados iguales 2 a $2 \ldots$

El uso conjunto por el grupo de estudiantes de las rutinas Identificar relaciones implicativas y Poner ejemplos va a producir un cambio en el discurso del grupo que lleva a una nueva narrativa asumida, Listado minimo de caracteristicas. Una evidencia empírica del uso de la rutina Identificar relaciones implicativas se muestra en el protocolo 3.2, donde se observa que a partir de características entre lados y ángulos se establecen características de los ángulos de las diagonales.

Protocolo 3.2:

52 A2: por ejemplo en la figura 1

53 A1: observamos que para ser un cuadrado tiene que tener los 3 lados iguales

54 A2: $\operatorname{los} 4$

$55 \mathrm{~A} 1: \operatorname{los} 4$

56 A2: y por tanto los mismos ángulos

$57 \mathrm{A1}$ : y sus 4 ángulos. Que las diagonales, el punto donde se cortan da lugar...

58 A2: esto implica...

59 A1: que donde se cruzan forman 4 ángulos iguales

Además, el grupo hace uso de la rutina Poner ejemplos, ya que como se puede observar en el protocolo 3.3 los estudiantes están tratando de poner un ejemplo, apoyándose en dibujos (mediadores visuales), para ver si alguna de las definiciones que han dado de las figuras puede incluir a otra figura distinta. Para ello, utilizan las características anteriormente descritas por ellos en la tarea y comprueban si el ejemplo (dibujo) que han propuesto cumple o no dichas características, introduciendo de esta forma nuevos mediadores visuales (dibujos de trapecios).

Protocolo 3.3:

225 A2: ¿hay alguna característica o propiedad común a las tres figuras?

226 A1: no, porque tienen una característica que las diferencia entonces...

227 A2: pero, ¿entre estas 3 figuras o entre otras figuras diferentes?

228 A1: no, entre estas 3

229 A2 hace un dibujo [dibujo de un trapecio], y comenta:

230 A2: por ejemplo, esto es un cuadrilátero, y esto tiene los ángulos iguales 2 a 2

231 A1: pero no son paralelos 2 a 2

232 A2: este con este sí es paralelo, y este con este sí es paralelo

$233 \mathrm{~A} 1$ : esto no es paralelo, los paralelos no se cruzan nunca

234 A2: es verdad, es verdad, pero seguro que alguno hay

El uso conjunto de estas dos rutinas facilita el cambio en el discurso, ya que les permite eliminar características de las figuras que se deducen de otras y comprobar el alcance de esa eliminación, tendiendo así a elaborar una definición con el atributo de la minimalidad, asumiendo el grupo de estudiantes una nueva narrativa Listado mínimo de características. Una evidencia empírica de este hecho la tenemos en el protocolo 3.4, cuando el grupo de estudiantes da una definición minimal que engloba dos figuras, cuadrado y rombo: 
Protocolo 3.4:

238 A1: si podemos dar una definición, por ejemplo del cuadrado, que incluya otra figura 239 A2: no puede ser

240 A1: no, pero... yo creo que de estas 3 figuras... que si podemos dar una definición que

241 sirva tanto para este como para este. Por ejemplo estas 2 figuras [refiriéndose al cuadrado

242 y al rombo]

243 A1: polígono cuadrilátero, lados iguales paralelos, diagonales, forman entre sí 4 ángulos

244 de $90^{\circ}$

En la segunda etapa, asumido por el grupo que para definir es suficiente con dar un listado mínimo de características y no es necesario un listado exhaustivo de ellas, el grupo asume una nueva narrativa, que es posible dar dos definiciones distintas del mismo objeto a través del uso de características distintas. Así, el grupo empieza a dar definiciones equivalentes de las mismas figuras, haciendo uso de las características de las diagonales de estas (líneas 215-216 [cuadrado]; líneas 218-219 [rectángulo]; líneas 221-224 [rombo], protocolo 3.5), en lugar de usar las propiedades de los lados de dichas figuras, como había hecho anteriormente. De esta manera, el grupo asume una nueva narrativa: «un objeto puede tener definiciones equivalentes» (narrativa Definiciones equivalentes), sin producirse el rechazo de la narrativa Listado mínimo de características. Es decir, asumir esta nueva narrativa no los lleva a rechazar la anterior, sino a aceptar la coexistencia de ambas.

Protocolo 3.5:

208 A1: ¿podrías dar otra definición?

209 A2: se trata de un cuadrilátero

210 A1: ahora podíamos hacerlo con las diagonales

.......

215 A2: polígono cuadrilátero cuyas 2 diagonales son iguales y entre ellos forman $90^{\circ}$, ya

216 está

217 A1: escribe

218 A2: rectángulo es un cuadrilátero cuyas diagonales son iguales y forman entre ellos

219 ángulos iguales 2 a 2

220 A1: rombo

221 A2: rombo se trata de un cuadrilátero cuyas diagonales son

$222 \mathrm{~A} 1$ : son distintas y

223 A2: forman $90^{\circ}$

$224 \mathrm{~A} 1$ : forman entre ellas ángulos de $90^{\circ}$

225 A1 lee la siguiente pregunta

226 no, porque tienen una característica que las diferencia entonces...

227 A2: pero, ¿entre estas 3 figuras o entre otras figuras diferentes?

228 A1: no, entre estas 3

229 A2 hace un dibujo, y comenta:

230 A2: por ejemplo, esto es un cuadrilátero, y esto tiene los ángulos iguales 2 a 2

231 A1: pero no son paralelos 2 a 2

232 A2: este con este sí es paralelo, y este con este sí es paralelo

233 A1: esto no es paralelo, los paralelos no se cruzan nunca

234 A2: es verdad, es verdad, pero seguro que alguno hay

235 Pasan a otra pregunta

236 A1: definición de una figura que incluya otra figura

$237 \mathrm{~A} 2$ : venga, venga

$238 \mathrm{~A} 1$ : sí podemos dar una definición, por ejemplo del cuadrado que incluya otra figura

239 A2: no puede ser

240 A1: no, pero... yo creo que de estas 3 figuras... que si podemos dar una definición que 
241 sirva tanto para este como para este. Por ejemplo estas 2 figuras...

242 A1: polígono cuadrilátero, lados iguales paralelos, diagonales forman entre sí 4 ángulos

243 de $90^{\circ}$

La aceptación de esta nueva narrativa se produce a través del uso de la rutina «Identificar relaciones (equivalentes) entre características de los objetos» (rutina Identificar relaciones equivalentes), que se ha puesto de manifiesto desde el principio en el discurso de este grupo (protocolo 3.6, líneas 57-59; 6771; 84-86; 89-90), donde se observa que se están estableciendo relaciones entre características de lados (longitudes y ángulos) con las características de diagonales (longitudes y ángulos) de las figuras.

Protocolo 3.6:

53 A1: observamos que para ser un cuadrado tiene que tener los 3 lados iguales

$54 \mathrm{~A} 2: \operatorname{los} 4$

$55 \mathrm{~A} 1: \operatorname{los} 4$

56 A2: y por tanto los mismos ángulos

$57 \mathrm{A1}$ : y sus 4 ángulos. Que las diagonales, el punto donde se cortan da lugar...

58 A2: esto implica...

59 A1: que donde se cruzan forman 4 ángulos iguales

.......

65 A2: son paralelos, ¿qué pongo?, es un rectángulo por lo que tiene sus lados

$66 \mathrm{~A} 1$ : iguales 2 a 2

67 A2: y paralelos, ¿qué más?, que el ángulo que se forma al cortar sus diagonales

68 A1: los ángulos también son iguales 2 a 2 . Los ángulos que forman los lados son rectos,

69 pero los ángulos que forman las diagonales...

70 A2: al cortarse

71 A1: forman ángulos iguales 2 a 2, este es igual que este, y este es igual que este, ¿qué 72 más?

.......

84 A2: claro, en el cuadrado los lados son iguales y las diagonales son iguales también, 85 pero en el rombo los lados son iguales pero las diagonales no son iguales, los ángulos 86 también, ¿no?

87 A1: los ángulos 2 a 2, este con este y este con este. Podemos poner las letras si no se 88 entiende

89 A2: al trazar las 2 posibles diagonales se comprueba que no miden lo mismo pero los

90 ángulos que forman al cortarse miden $90^{\circ}$

En el discurso del grupo de estudiantes se hace evidente que existen relaciones de equivalencia (doble implicación) entre las características de cada una de las figuras, lo que los lleva a poder dar definiciones equivalentes. Así, las figuras definidas en un principio a través de lados y ángulos comprendidos entre ellos pasan a ser definidas a través de diagonales y ángulos formados por estas. Además, introducen otros mediadores visuales de tipo simbólico, ya que señalan que para referirse a un elemento, este puede nombrarse de forma simbólica (letras) (líneas 87 y 88 del protocolo 3.6).

En resumen, en el discurso matemático de este grupo hemos identificado cambios en el discurso en dos etapas, caracterizados por cambios en narrativas asumidas por el grupo, a través del empleo de determinadas rutinas, del uso de determinadas palabras matemáticas y de la producción de nuevos mediadores visuales. Los cambios en las narrativas asumidas, en algunas ocasiones, no conllevan el abandono o rechazo de las previamente asumidas por el grupo, característica relevante de esta modalidad de cambio en el discurso. De esta manera, a diferencia de otras situaciones, el grupo puede hacer uso de las distintas narrativas que han sido asumidas por este en diferentes momentos del proceso discursivo. 


\section{CONCLUSIONES}

En este trabajo, hemos caracterizado los procedimientos de cambios en el discurso matemático de grupos de estudiantes a través de las herramientas teóricas (palabras matemáticas, mediadores visuales, rutinas y narrativas asumidas) cuando resuelven una tarea sobre el contenido matemático de definir/ definición, en un contexto geométrico.

Con relación al proceso de definir en el contexto geométrico, los resultados muestran que determinadas rutinas juegan un papel relevante en los procedimientos de cambios que se han producido en el discurso matemático. Por ejemplo, en el cambio de la narrativa Etiquetar a la narrativa Listado de características es fundamental el uso de la rutina Identificar caracteristicas. Del mismo modo, en los cambios en el discurso matemático que se producen para llegar a la narrativa Listado mínimo de características, hacer uso de las rutinas Identificar relaciones implicativas y Poner ejemplos es clave para dichos cambios. Asimismo, para aceptar la narrativa Definiciones equivalentes es decisivo utilizar la rutina Identificar relaciones equivalentes.

En nuestros resultados se pone de manifiesto que, en los cambios en el discurso, se usan rutinas que centran su atención en las características de los objetos que se están definiendo y rutinas cuyo foco de atención son las relaciones entre las características de los objetos que se están definiendo. Estas diferencias en el foco de atención de las rutinas son esenciales para diferenciar las distintas formas de considerar (conceptualizar) el proceso de definir de los estudiantes. Así, los grupos de estudiantes que centran su atención en las características de los objetos, haciendo uso solo de la rutina Identificar caracteristicas, son grupos que consideran solo definiciones dadas por un conjunto de características que pueden ser redundantes, es decir, para ellos cada objeto tiene una única definición. Mientras que otros grupos de estudiantes, al hacer uso de otras rutinas, como Identificar relaciones implicativas o Identificar relaciones equivalentes, consideran definiciones matemáticas que pueden ser válidas para varios objetos que se están definiendo o definiciones diferentes para un mismo objeto.

En este sentido, nuestros resultados son coincidentes con los obtenidos por Güçler (2013), respecto a que las narrativas asumidas pueden describir cómo se comparten los significados de los conceptos matemáticos en comunidades de prácticas y cómo se producen los cambios en los significados de dichos conceptos matemáticos, esencialmente a través del uso de rutinas.

Por otro lado, en cuanto al aprendizaje producido en los grupos de estudiantes en trabajos previos (Escudero et al., en prensa), identificamos diferentes cambios en el discurso. A partir de esta identificación, mediante un proceso de análisis inductivo sobre las características de los procedimientos de cambio, en términos de las herramientas teóricas de la perspectiva sociocultural de Sfard (palabras matemáticas, mediadores visuales, narrativas asumidas y rutinas), en este trabajo hemos identificado diferentes situaciones de aprendizaje matemático en el proceso de definir:

1. Situación de invariancia en el discurso matemático en definir: caracterizado por la invariancia en el discurso matemático, es decir, en los grupos de estudiantes no se producen cambios en las herramientas discursivas (palabras matemáticas, narrativas asumidas, rutinas y mediadores visuales).

2. Situación de un cambio discursivo en definir con abandono de narrativa: caracterizado por un cambio en el discurso matemático de una narrativa asumida a otra distinta. La aceptación de la nueva narrativa conlleva el rechazo de la primera. Este cambio en el discurso, aunque caracterizado por el uso de determinadas rutinas, también conlleva cambios en el significado de determinadas palabras matemáticas y el uso de nuevos mediadores visuales.

3. Situación de varios cambios discursivos secuenciados en definir, con abandono de narrativas: caracterizado por cambios en el discurso matemático, del tipo de los identificados en la situación 
anterior, de al menos dos etapas, de tal forma que la aceptación de una nueva narrativa, en cada etapa, conlleva el rechazo de la anterior.

4. Situación de varios cambios discursivos coordinados en definir: caracterizado por cambios en el discurso, en varias etapas, donde los cambios de narrativas no implican el rechazo de otras. Esta situación se caracteriza por que los grupos disponen de un repertorio de varias narrativas asumidas con las que afrontar situaciones problemáticas.

Aunque en nuestra investigación no tenemos evidencia empírica de que en los cambios en el discurso matemático en una sola etapa («Situación de un cambio discursivo en definir con abandono de narrativa») se pueda producir la coexistencia de dos narrativas, pensamos que esta posibilidad puede darse. Esto es una limitación de nuestro estudio, ya que está centrado en un contexto geométrico específico y utiliza una única tarea. En este sentido, sería conveniente confirmar en futuras investigaciones los diferentes cambios discursivos observados en los grupos, a través del uso de diferentes tareas, en distintas sesiones de clase.

Este trabajo puede continuarse abriendo futuras líneas de investigación vinculadas a otros procesos matemáticos, tales como probar/demostrar, modelar, o a otros tópicos matemáticos.

\section{AGRADECIMIENTOS}

A los revisores anónimos cuyos comentarios y sugerencias han permitido mejorar este trabajo. Este trabajo ha sido posible gracias al apoyo del proyecto de investigación PSI2008-02289 financiado por el Ministerio de Ciencia e Innovación.

\section{REFERENCIAS}

Bowers, J.S. y STEPHEns, B. (2011). Using technology to explore mathematical relationships: a framework for orienting mathematics courses for prospective teachers. Journal of Mathematics Teacher Education, 14, pp. 285-304.

http://dx.doi.org/10.1007/s10857-011-9168-x

Chico, J. y Planas, N. (2011). Interpretación de indicadores discursivos en situaciones de aprendizaje matemático en pareja. En M. Marín, G. Fernández, L.J. Blanco y M. Palarea (eds.). Actas del XV Simposio de la Sociedad Española de Investigación en Educación Matemática. Ciudad Real, España: SEIEM, pp. 319-328.

Cobb, P.; Gresalfi, M. y Liao Hodge, L. (2009). An Interpretive Scheme for analyzing the Identities that Students Develop in Mathematics Classrooms. Journal for Research in Mathematics Education, 40, pp. 40-68.

Сobo, P. y Fortuny, J.M. (2000). Social Interactions and Cognitive Effects in Contexts of AreaComparison Problem Solving. Educational Studies in Mathematics, 42, pp. 115-140. http://dx.doi.org/10.1023/A:1004187711956

Escudero, I.; Gavilán, J.M. y Sánchez-Matamoros, G. (en prensa). Una aproximación a los cambios en el discurso matemático generados en el proceso de definir. Revista Latinoamericana de Investigación en Matemática Educativa.

García, M.; SÁnchez, V. y Escudero, I. (2007). Learning through reflection in mathematics teacher education. Educational Studies in Mathematics, 64, pp. 1-17.

García, M.; Sánchez, V.; Escudero, I. y Llinares, S. (2003). The dialectic relationship between theory and practice in Mathematics Teacher Education. En M.A. Mariotti (ed.). Proceedings of the III European Research In Mathematics Education. Bellaria, Italy: ERME. 
http://dx.doi.org/10.1007/s10649-006-9021-9

Gavilán, J.M.; García, M. y Llinares, S. (2007). Una perspectiva para el análisis de la práctica del profesor de matemáticas. Implicaciones metodológicas. Enseñanza de las Ciencias, 25 (2), pp. 157 170.

Gavilán, J.M.; Sánchez-Matamoros, G. y Escudero, I. (2012). El proceso de definir en matemáticas desde una perspectiva comognitiva. En A. Estepa, A. Contreras, J. Deulofeu, M.C. Penalva, F.J. García y L. Ordoñez (eds.). Actas del XVI Simposio de la Sociedad Española de Investigación en Educación Matemática. Jaén, Espańa: SEIEM, pp. 275-283.

GüçLER, B. (2013). Examining the discourse on the limit concept in a beginning-level calculus classroom. Educational Studies in Mathematics, 82, pp. 439-453.

http://dx.doi.org/10.1007/s10649-012-9438-2

Hershkowitz, R. (1990). Psychological aspects of learning geometry. En P. Nesher y J. Kilpatrick (eds.). Mathematics and Cognition. Cambridge, UK: Cambridge University Press, pp. 70-95.

Lave, J. y Wenger, E. (1991). Situated Learning. Legitimate Peripheral Participation. Cambridge, UK: Cambridge University Press. http://dx.doi.org/10.1017/CBO9780511815355

LERMAN, S. (1996). Intersubjectivity in mathematics learning: A challenge to the radical constructivist paradigm? Journal for Research in Mathematics Education, 27, pp. 133-150. http://dx.doi.org/10.2307/749597

Lerman, S. (2000). The social turn in mathematics education research. En J. Boaler (ed.). Multiple perspectives on mathematics teaching and learning. Westport, CT, USA: Ablex Publishing, pp. 19-44.

Lerman, S. (2006). Cultural psychology, anthropology and sociology: the developing «strong», social turn. En J. Maasz y W. Schloeglmann (coords.). New mathematics education research and practice. Rotterdam, Holanda: Sense Publishers, pp. 171-188.

Llinares, S. (2000). Comprendiendo la práctica del profesor de matemáticas. En J.P. Ponte y L. Sarrazina (eds.). Educaçao Matemática em Portugal, Espanha e Italia, Actas da Escola de Verao-1999. Lisboa, Portugal: Sección de Educación Matemática Sociedad Portuguesa de Ciencias de la Educación/Sociedad de Educación y Matemática, pp. 109-132.

Mamona-Downs, J. y Downs, M. (2002). Advanced Mathematical Thinking With a Special Reference to Reflection on Mathematical Structure. En L.D. English (ed.). Handbook of International Research in Mathematics Education. Mahwah, NY, USA: Lawrence Erlbaum, pp. 165-195.

Mariotti, M.A. y Fischbein, E. (1997). Defining in classroom activities. Educational Studies in Mathematics, 34, pp. 219-248. http://dx.doi.org/10.1023/A:1002985109323

Morera, L.; Planas, N. y Fortuny, J.M. (2013). Design and validation of a tool for the analysis of whole group discussions in the mathematics classroom. En B. Ubuz et al. (eds.). Proceedings of the VIII Congress of European Research in Mathematics Education. Antalya, Turquía: ERME.

Planas, N. y Chico, J. (2013). The productive role of interaction: Students' algebraic thinking in whole group discussion. En B. Ubuz et al. (eds.). Proceedings of the VIII Congress of European Research in Mathematics Education. Antalya, Turquía: ERME.

Planas, N. y Civil, M. (2010). Discourse processes in critical mathematics education. En H. Alrø, O. Ravn y P. Valero (eds.). Critical mathematics education: past, present and future. Festschrift for Ole Skovsmose. Rotterdam, Holanda: Sense Publishers, pp. 145-160.

Rasmussen, C.; Zandieh, M.; King, K. y Teppo, A. (2005). Advancing Mathematical Activity: A Practice-Oriented View of Advanced Mathematical Thinking. Mathematical thinking and learning, 7, pp. 51-73.

http://dx.doi.org/10.1207/s15327833mt10701_4 
Sánchez, V. y García, M. (2011). Socio-Mathematical and Mathematical norms in pre-service primary teachers' discourse. En B. Ubuz (ed.). Proceedings of the 35th PME International Conference, vol. 4. Ankara, Turkey: PME, pp. 105-112.

SÁnchez, V. y García, M. (2014). Sociomathematical and mathematical norms related to definition in pre-service primary teachers' discourse. Educational Studies in Mathematics, 85, 305-320. http://dx.doi.org/10.1007/s10649-013-9516-0

Sánchez, V.; García, M.; Escudero, I.; Gavilán, J.M. y Sánchez-Matamoros, G. (2008). Una aproximación a las matemáticas en el bachillerato. ¿Qué se pretende que aprendan los alumnos? Enseñanza de las Ciencias, 26 (2), pp. 267-276.

Sánchez-Matamoros, G.; Escudero, I. y Gavilán, J.M. (2011). Defining from sociocultural approach. En B. Ubuz (ed.). Proceedings of the 35th PME International Conference, vol. 1. Ankara, Turkey: PME, p. 500.

SFARD, A. (2006). Participacionist discourse on mathematics learning. En J. Maasz y W. Schlöglmann (eds.). New Mathematics Education Research and Practice. Rotterdam, Holanda: Sense Publishers, pp. 153-170.

SFARD, A. (2007). When the Rules of Discourse Change, but Nobody Tells You: Making Sense of Mathematics Learning From a Commognitive Standpoint. Journal of the Learning Sciences, 16, pp. 565-613. http://dx.doi.org/10.1080/10508400701525253

SFARD, A. (2008). Thinking as communicating: human development, the growth of discourse, and mathematizing. Cambridge, UK: Cambridge University Press. http://dx.doi.org/10.1017/CBO9780511499944

TAll, D. y Vinner, S. (1981). Concept Image and Concept Definition in Mathematics with particular reference to limits and continuity. Educational Studies in Mathematics, 12, pp. 151-169. http://dx.doi.org/10.1007/BF00305619

Vinner, S. y Hershkowitz, R. (1980). Concept images and common cognitive paths in the development of some simple geometrical concepts. En R. Karplus (ed.). Proceedings of the 4th PME International Conference. Berkeley, USA: PME, pp. 177-184.

VygostKi, L.S. (1962). Thought and language (traducción de Eugenia Hanfmann y Gertrude Vakar). New York (USA) and London, UK: Massachusetts Institute of Technology and John Wiley and sons.

Wenger, E. (1998). Communities of practice. New York, USA: Cambridge University Press. http://dx.doi.org/10.1017/CBO9780511803932

Zandieh, M. y Rasmussen, C. (2010). Defining as a mathematical activity: A framework for characterizing progress from informal to more formal ways of reasoning. Journal of Mathematical Behavior, 29, pp. 57-75. http://dx.doi.org/10.1016/j.jmathb.2010.01.001

ZAZKIs, R. y Leikin, R. (2008). Exemplifying definitions: a case of a square. Educational Studies in Mathematics, 69, pp. 131-148.

http://dx.doi.org/10.1007/s10649-008-9131-7 
Aprender a definir en Matemáticas: estudio desde una perspectiva sociocultural 


\title{
Learning to define in Mathematics: a study from a sociocultural perspective
}

\author{
José María Gavilán Izquierdo, Gloria Sánchez-Matamoros, Isabel Escudero \\ Departamento de Didáctica de las Matemáticas \\ Universidad de Sevilla \\ gavilan@us.es, gsanchezmatamoros@us.es, escudero@us.es
}

The process of building mathematical knowledge, as defined, is the key activity of "doing mathematics". The characterization of learning is a relevant topic in the field of research in mathematics education. In this paper, we consider "defining" as the process to get to state a definition, considering that the latter prescribes the meaning of a word or phrase specifically in terms of a list of features that must all be true (Sánchez et al., 2008). In the sociocultural perspective, adopted mathematical learning means changing mathematical discourse. Sfard (2007, 2008) proposed the use of four discursive tools to characterize the mathematical discourse: "mathematical words, such as those related to quantities and shapes; visual mediators are means with which participants of discourses identify the object of their talk and coordinate their communication; routines are well-defined repetitive patterns in interlocutors' actions, characteristic of a given discourse, and narrative is any text, spoken or written, that is framed as a description of objects, or of relations between objects or activities with or by objects, and that is subject to endorsement or rejection." (Sfard, 2007, pp 573-574).

Through an inductive analysis, we identified different situations of learning: a) Situation of invariance in mathematical discourse in defining, b) Situation of change in mathematical discourse with no narrative, c) Situation of various changes in the mathematical discourse sequenced to define, with no narrative, and d) Situation sequenced with several changes in the mathematical discourse to define.

Our results show two types of routines in the changes of discourse: the routines that focus on the characteristics of the objects that are being defined, and the ones that focus on the relationship between the characteristics of the objects that are being defined. These differences in the focus of the routines are key to differentiate the various approaches to define (conceptualize) the students' process. 